\title{
Primary hepatic neuroendocrine tumor
}

\author{
Le Tuan Linh ${ }^{1,2, *}$, Nguyen Minh Duc ${ }^{(1,4, *}$, Hoang Tu Minh${ }^{2}$, Nguyen Ngoc Cuong', Vuong Thu Ha1, \\ Dao-Thi Luan ${ }^{5}$, Thieu-Thi Tra My² and Bui Van Lenh ${ }^{1,2}$
}

${ }^{1}$ Department of Radiology, Hanoi Medical University Hospital, Hanoi, Vietnam, 2Department of Radiology, Hanoi Medical University, Hanoi, Vietnam, ${ }^{3}$ Department of Radiology, Pham Ngoc Thach University of Medicine, Ho Chi Minh city, Vietnam, 4Department of Radiology, Childrent's Hospital 2, Ho Chi Minh city, Vietnam, and

${ }^{5}$ Department of Pathology, Hanoi Medical University Hospital, Hanoi, Vietnam

*(L T Linh and N M Duc contributed equally to this work)

Correspondence should be addressed to N M Duc Email

bsnguyenminhduc@ pnt.edu.vn

\section{Summary}

Primary hepatic neuroendocrine tumor (PHNET) is a rare type of neuroendocrine tumor (NET) that is also a primary hepatic tumor. Patients are present with almost no specific clinical symptoms and typically present with negative test results and atypical imaging characteristics; therefore, the differentiation of PHNET from other types of primary hepatic masses can be very difficult. In this article, we describe a case of PHNET that mimicked a liver helminth infection in a 57-year-old man. The diagnosis of PHNET in this patient was challenging, and the final diagnosis was based on imaging, histopathology features, and long-term follow-up.

\section{Learning points:}

- An uncommon type of neuroendocrine tumor (NET) is a primary hepatic neuroendocrine tumor (PHNET).

- Primary hepatic neuroendocrine tumors are rare NET lesions found in the liver, characterized by non-specific clinical and imaging results, which can be easily confused with other liver lesions, including HCC and parasitic lesions.

- To have a conclusive diagnosis and classification, a mixture of many medical assessment techniques, such as imaging, gastrointestinal endoscopy, nuclear medicine, anatomy, including histopathology, and immunohistochemistry, is essential.

\section{Background}

Neuroendocrine tumors (NETs) are tumors that originate from the cells of the neuroendocrine system (1). NETs can be observed in many important organs within the human body, with approximately $50 \%$ of NETs occurring in the gastrointestinal tract and approximately $30 \%$ occurring in the bronchopulmonary tree (2). NETs are rarely described in the liver, and those identified in the liver are typically the result of metastasis from other organs (2). Primary hepatic NET (PHNET) is an extremely rare hepatic lesion (3). The first report of this disease was described by Edmondson in 1958. According to a review of the literature, as reported by Li et al. (4), only approximately 150 cases of PHNET have been reported. In most cases, lesions occur in adults, with no apparent sex predilection, presenting as a solid mass (5). PHNETs have no specific imaging features and are typically misdiagnosed as other hepatic lesions such as hepatocellular carcinoma (HCC) or cholangiocarcinoma (CCC) (2). The pathological results of PHNETs do not differ from those of other NETs (6). However, neither imaging studies nor pathology studies are able to differentiate a primary PHNET lesion from a secondary metastatic NET; therefore, the diagnosis of PHNET is particularly concerning (7). 


\section{Case report}

A 57-year-old man was admitted to Hanoi Medical University Hospital complaining of abdominal pain in the lower quadrant and diarrhea. Abdominal ultrasound images showed a well-defined nodule, $20 \mathrm{~mm}$ in diameter, with a heterogeneous hyperechoic and hypoechoic border, involving segment VII of the liver.

\section{Investigation}

The patient underwent an MRI scan of the upper abdomen, using a Signa HD× 1.5 Tesla, with the injection of $10 \mathrm{~mL}$ Gadoteric acid (Dotarem, Guerbert, France, a dose of $0.5 \mathrm{mmol} / \mathrm{mL}, \times 10 \mathrm{~mL}$ ). MRI revealed two well-defined and lobulated nodules in segments IV and VII, with dimensions of $22 \times 28 \mathrm{~mm}$ and $15 \times 5 \mathrm{~mm}$, respectively. These lesions increased in intensity on T2-weighted image, decreased in intensity on T1-weighted image, and were hyperintense on the diffusion-weighted image (DWI, Fig. 1). The lesions were presented with heterogeneous enhancement on arterial and venous phase images, with primarily peripheral enhancement (Fig. 2). These findings were consistent with lesions caused by parasites.

The blood test showed complete blood cell counts, alpha-fetoprotein (AFP), carcinoembryonic antigen (CEA), and carbohydrate antigen 19.9 (CA19.9) levels within the normal range, and high-sensitivity C-reactive protein (hs-CRP) at $0.19 \mathrm{mg} / \mathrm{dL}$ (normal range is $<0.3 \mathrm{mg} /$ dL). Hepatitis B surface antigen (HBsAg) and hepatitis $\mathrm{C}$ virus antibody ( $\mathrm{HCV} \mathrm{Ab}$ ) were negative. Enzymelinked immunosorbent assays (ELISAs) were performed for parasitic antigens, including Entamoeba histolytica, Clonorchis, Echinococcus, Fasciola, Strongyloides stercoralis, and Toxocara immunoglobulin G (IgG), which revealed positive results for Clonorchis $\operatorname{IgG}(0.177 \mathrm{ng} / \mathrm{mL})$ and Toxocara IgG $(0.578 \mathrm{ng} / \mathrm{mL})$. The patient was treated with Albendazole $200 \mathrm{mg} \times$ four pills/day over 2 weeks. After
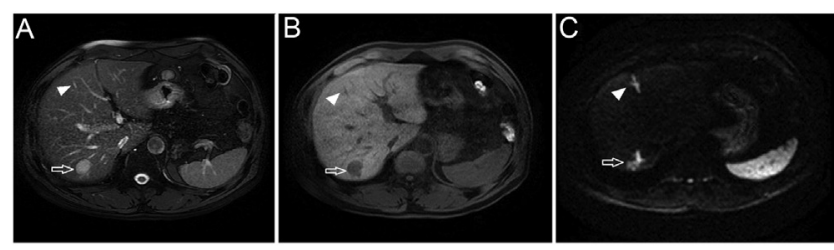

\section{Figure 1}

Upper abdominal MRI revealed lesions in the VII segment (white arrow) and IV segment (white arrowhead) of the liver. (A) Hyperintense lesions on T2-weighted image. (B) Hypointense lesions on T1-weighted image. (C) Lesions were hyperintense on diffusion-weighted image.

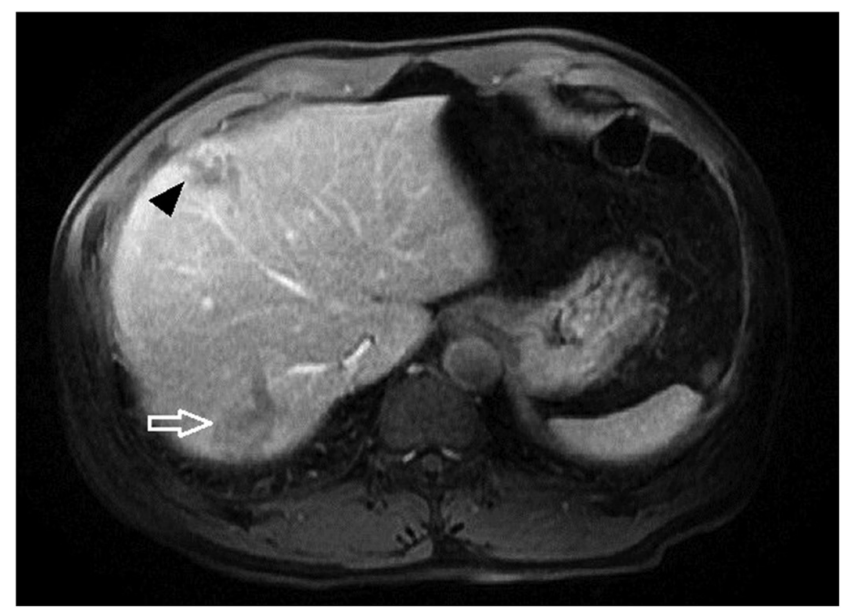

\section{Figure 2}

The lesions were heterogeneous enhancement (white arrow and black arrowhead).

4 months, the patient was presented to the hospital due to recurrent abdominal pain and diarrhea. Ultrasound images revealed the marked progression in the sizes of the two previously identified lesions, with the mass in segment VII measuring at $38 \times 32 \mathrm{~mm}$ and the mass in segment IV measuring at $15 \times 15 \mathrm{~mm}$. Both masses presented with hyperechoic and hypoechoic border characteristics, similar to the initial evaluation (Fig. 3).

On abdominal MRI, the sizes of the masses identified in segment VII and IV were $40 \times 31 \mathrm{~mm}$ and $15 \times 23 \mathrm{~mm}$,

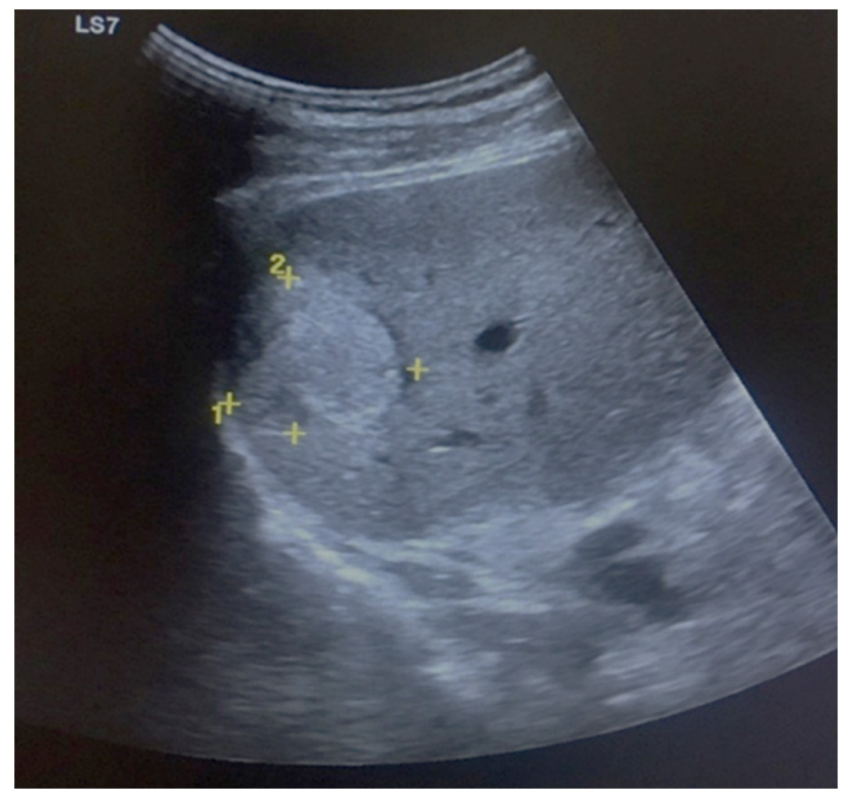

Figure 3

The second abdominal ultrasound. Well-defined hyperechoic lesion with and hypoechoic border identified the seventh segment of the liver. 
respectively, which were both larger than measured during the first evaluation.

These lesions increased intensity on T2-weighted image, decreased intensity on T1-weighted image (Fig. 4), restricted diffusion by showing hyperintense on diffusion-weighted image and hypointense on apparent diffusion coefficient map (Fig. 5), poor enhancement on arterial phase images without washout sign on the portal phase or delayed phase images (Fig. 6). These features were similar to the first evaluation.

Chest X-rays showed no abnormal findings. Colonoscopy revealed proctitis with erythematous swelling. A rectal endoscopic biopsy was planned to confirm the diagnosis, and the pathological results suggested chronic inflammation. The stool test revealed no presence of worm larvae or red blood cells. Other blood test results, such as marker AFP, CEA, CA199, total prostate-specific antigen (PSA), white blood cell percentage, CRP, and parasitic ELISAs (Entamoeba histolytica, Clonorchis, Echinococcus, Fasciola, Strongyloides stercoralis, and Toxocara IgG) were within the normal range. The significant progression in the sizes of these two hepatic masses led the clinicians to obtain a biopsy of the lesion in segment VII. The pathological findings suggested a glandular structure, with a large, round or oval nucleus and no mitotic nuclear or necrosis (Fig. 7). Immunohistochemistry revealed positive staining for clusters of differentiation 56 (CD56), synaptophysin, $\mathrm{P} 53$, and cytokeratin (CK). The tumor cells were negative for chromophysin, and the tumor had a Ki-67 index of 20\% (Fig. 8).

Then, the patient was submitted to a whole-body PET and CT (PET-CT) scan using 18F-fluorodeoxyglucose (18F-FDG). Images revealed two hypometabolic hepatic
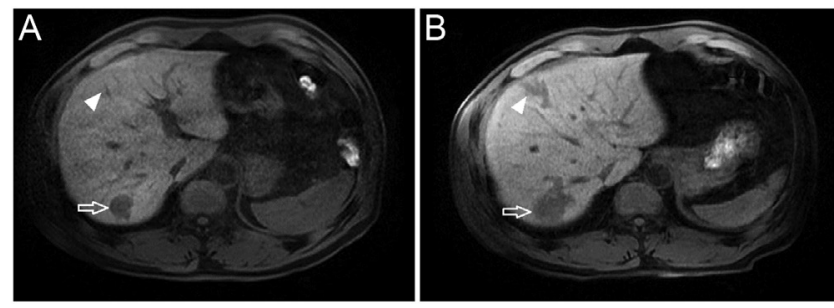

\section{Figure 4}

(A) First abdominal MRI result: T1-weighted, fat-suppressed image showing a $22 \times 28 \mathrm{~mm}$ nodule in segment VII of the liver (white arrow) and a small, faint lesion in segment IV (white arrowhead). (B) Second abdominal MRI images: T1-weighted, fat-suppressed imaging revealed that liver lesions increased in size after 4 months, with the segment VII mass measuring at $40 \times 31 \mathrm{~mm}$ (white arrow) and the lesion in segment IV clearly visible and $15 \times 23 \mathrm{~mm}$ in size (white arrowhead).
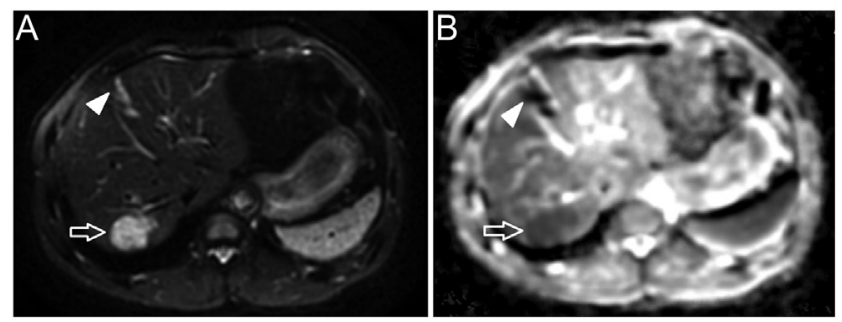

\section{Figure 5}

Second abdominal MRI images, two lesions (white arrow and white arrowhead) showed restricted diffusion. (A) Lesions were hyperintense on diffusion-weighted image. (B) Lesions were hypointense on apparent diffusion coefficient (ADC) map.

lesions, and no hypermetabolic lesions were identified in any other organs (Fig. 9).

Based on the MRI results, the normal levels of CEA, CA19-9 and AFP and other test results within the normal range, the histopathology results, and no findings of other extra-hepatic lesions, the diagnosis for the second evaluation was PHNET, grade 2 .

\section{Outcome and follow-up}

Surgical intervention was recommended, but the patient refused and was not re-evaluated until 1 year later due to a lack of abnormal symptoms. Fifteen months after the last check-up, the patient suffered abdominal pain in the right upper quadrant for 2 weeks, and he was admitted to the hospital. An abdominal CT (CT) scan, consisting of 64 slices, was performed using the contrast agent iopromide $0.625 \mathrm{~g} / \mathrm{mL}$ at $1 \mathrm{~mL} / \mathrm{kg}$ (Ultravist 300). The abdominal CT showed that the lesions in segment IV and VII had progressed dramatically, overtaking most of segment IV and the right liver. The lesions featured uneven, ill-defined borders, which were heterogeneous and hypoechoic, with a calcified component. Uneven high hypervascularity was observed in arterial phase images, and a washout sign was observed on the portal venous phase image, revealing that the center of the lesion featured poor contrast enhancement, suggesting necrosis. Furthermore, the imaging findings showed thrombosis in the total right branch and the partial left branch of the portal vein (Fig. 10). All of these characteristics were characteristic of hepatic lesions due to HCC. Chest CT scans showed no abnormal findings.

Blood tests were performed to examine the AFP, CEA, and CA19.9 levels, and the results were within the normal range. The colonoscopy was repeated, and no suspected lesions were identified. The final diagnosis was PHNETs. 

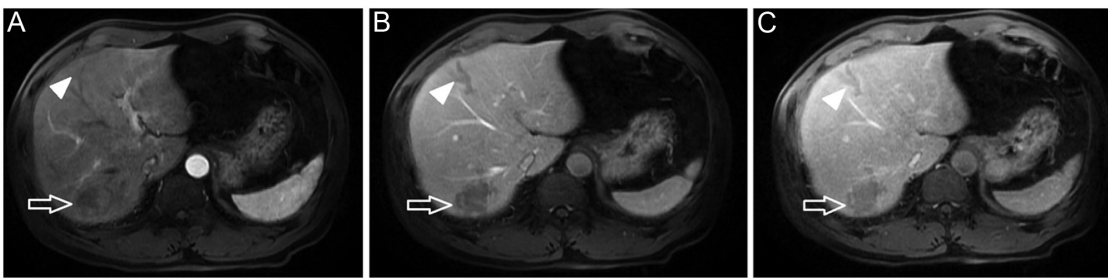

\section{Figure 6}

Second abdominal MRI images. (A) lesions were poorly enhancement on arterial phase. There was no washout sign of lesions on the portal phase (B) or delayed phase images (C).

\section{Discussion}

Neuroendocrine tumors develop from cells in the neuroendocrine system and can be located in many organs, with 54 to $90 \%$ of all NET cases arising from the gastrointestinal tract (2). Primary hepatic neuroendocrine tumors are very rare, and very little research has been performed examining the characteristics and treatment methods (3). NET lesions can be divided into two large groups: hormone-secreting tumors and non-hormone-secreting tumors. PHNETs are typically non-hormone-secreting tumors (8). The classification system used for NET classification was established by the World Health Organization (WHO) in 2010 and is based on the pathology findings, including the cell morphology, the number of mitotic cells identified in ten high-power fields of view, and the Ki67 index (1). Based on this classification system, NET tumors in the pancreas and gastrointestinal tract (GEP-NETs) can be classified into three types: low malignancy (grade 1), moderate malignancy (grade 2), and high malignancy (grade 3 ), with increasingly poor prognosis associated with ascending grade (1). Grade 3 NET is classified as neuroendocrine carcinoma (NEC), with poorly differentiated cells, including two small-cell types and one large-cell type (1).

To date, no classification system has been established for PHNET (2). However, PHNET can be classified

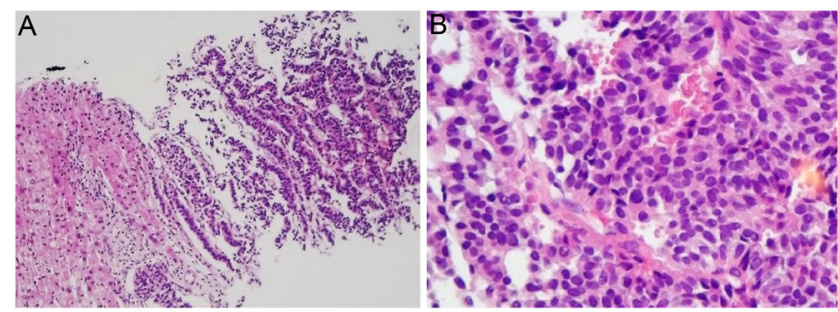

Figure 7

Histopathology: (A) Hematoxylin-eosin staining $\times 10$ magnification. Tumor tissue, bordered by normal liver tissue. The tumor-aligned cells suggested a glandular structure. (B) Hematoxylin-eosin staining $\times 100$ magnification. The tumor cells are uniform, with large, round, or oval nuclei, fine, granular chromatin, and lying to one side, with no abnormal mitotic activity or necrosis. according to the previously described NET classification, which is very useful for evaluating the prognosis and malignancy potential of tumors (9). The clinical manifestations of PHNET differ from those associated with other NETs. PHNET typically grows slowly and is not a hormone-secreting tumor; therefore, they are typically discovered only by chance and manifest in the clinic only during the late stage (10). Most PHNETs are solitary (76.6\%), but they can also be multifocal and are more common in the right lobe (46.8\%) (8). Our patient was a 57 -year-old man with two tumors in his liver that were identified by chance.

The diagnosis of PHNET involves a pre-operative to post-operative process, which typically includes long-term follow-up for the detection of primary extra-hepatic lesions (11). On imaging, PHNET is often misdiagnosed as HCC or cholangiocarcinoma (CCC) (2), and PHNET lesions are atypical, presenting as a mass that can be either nodular or cystic and either welldefined or not (5). As reported by Wang et al. (12), all PHNET lesions show arterial phase enhancement and can be confused with other hypervascular liver lesions, and images showing PHNET lesions causing portal thrombosis have been included in several reports (12). Huang et al. reported a patient with huge PHNET in the left lobe, portal vein tumor thrombus and elevated AFP (13). In this case, after more than 1 year since PHNET was detected, the development of the tumor produced portal venous thrombosis but the AFP value was within the normal range.

Pathology plays an important role in the diagnosis of PHNET (1), and the histopathology of PHNET is similar to that observed for other NET tumors, featuring masses containing a mixture of solid and cystic components, with or without necrotic bleeding on gross images (9). On microscopic images, the tumors tend to present a nested, trabecular, or microacinar architecture, composed of small, uniform tumor cells with granular chromatin, round nuclei, and often stromal hyalinization (6). On immunohistochemistry, NETs have been reported to be positive for the markers chromogranin, synaptophysin, neuron-specific enolase, and CD56 (14). 

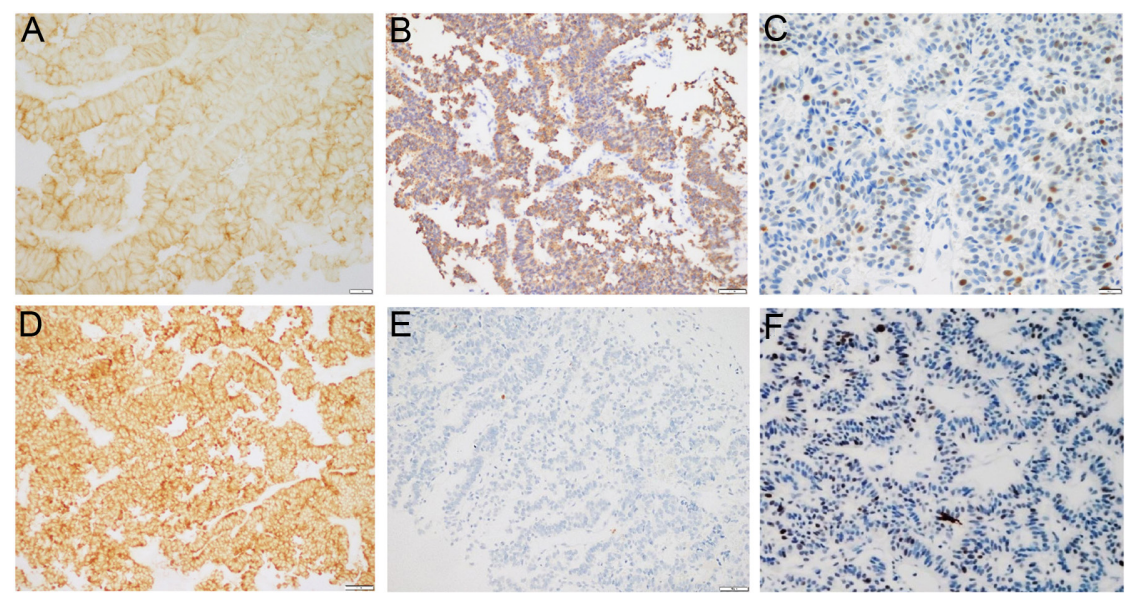

\section{Figure 8}

Immunohistochemistry $(A, \times 40)$ Tumor cells are positive for CD56. $(B, \times 20)$ Tumor cells are positive for CK marker. $(C, \times 40)$ Tumor cells are positive for marker P53. ( $\mathrm{D}, \times 20)$ synaptophysin-positive cells. $(E, \times 20)$ Tumor cells are negative with chromogranin marker. $(F, \times 40)$ Ki67 index $20 \%$.

The determination of the Ki67 index is used to evaluate the ratio of positive tumor nuclei (6). According to the WHO classification system, PHNET can be classified as grade 1 or grade 2 ; however, PHNET with grade 3 has not yet been reported (6). In most cases reported, PHNET was classified as grade 1 or grade 2 based on the WHO classification, however, Huang et al. reported a PHNET case with a huge liver tumor, and the pathology demonstrated poorly differentiated neuroendocrine carcinoma (NEC) (13) In this case, the lesions were positive for CK, synaptophysin, CD56, and P53, and negative for chromogranin marker, with a Ki67 index of $20 \%$. The histopathology and immunohistochemistry
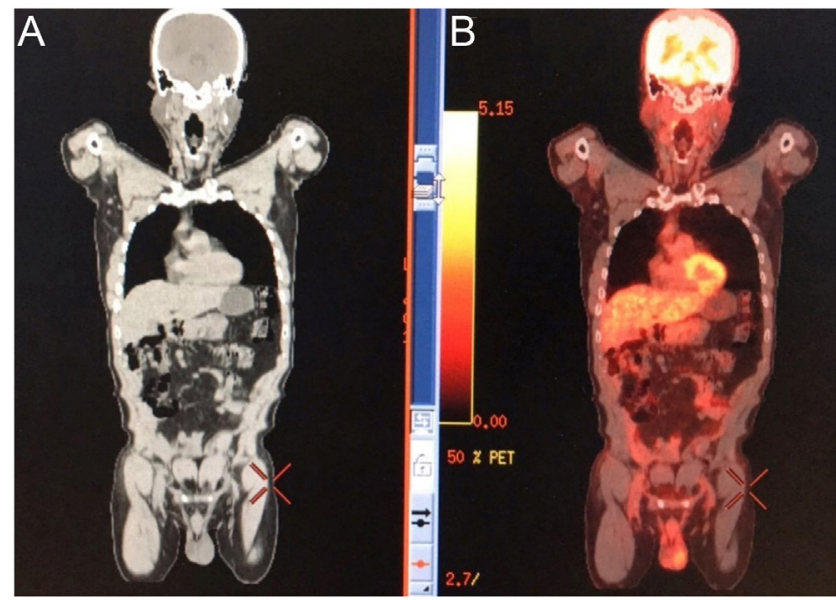

Figure 9

PET-CT with ${ }^{18} \mathrm{~F}-\mathrm{FDG}$. (A) CT and (B) PET-CT scans. Two hypometabolic hepatic lesions were observed, and no hypermetabolic lesions were identified in other organs. results for this patient were consistent with a diagnosis of grade 2 NET.

The imaging and pathological features associated with NET in the liver are unable to distinguish between primary PHNET or secondary tumors that result from metastasis from NETs in other tissues (7). Therefore, careful examinations must be performed to exclude the presence of extra-hepatic NET through the application of CT, MRI, somatostatin scans, PET, gastroscopy, colonoscopy, bronchoscopy, and surgical probes. Even after PHNET has been diagnosed, the long-term follow-up of patients to detect any potentially missed extra-hepatic lesion remains indispensable (2). In this case, our patient received two abdominal MRI exams, a colonoscopy, chest radiographs, and a systemic PET-CT scan, in addition to tests performed to exclude the potential existence of parasites. No NET lesions were detected outside of the liver, which resulted in the diagnosis of PHNET at the time of examination.

The application of PET-CT scans using ${ }^{18} \mathrm{~F}$-fluorodeoxyglucose (18F-FDG) for PHNET assessment has been described in a few reports around the world (15). The degree of ${ }^{18} \mathrm{~F}$-FDG uptake on PET-CT was proportional to the Ki67 index. Grade 1 NETs with low Ki67 index values typically display hypometabolism, whereas grade 2 NETs can present with either hypermetabolism or hypometabolism (15). In the patient in our study, PET-CT with ${ }^{18}$ F-FDG identified two lesions in the liver that were characterized by hypometabolism, and no other primary lesions were identified. However, the absence of observed metabolic damage on PET-CT scans, including liver lesions, cannot exclude the possibility of non-metabolic primary NET lesions outside the liver. 

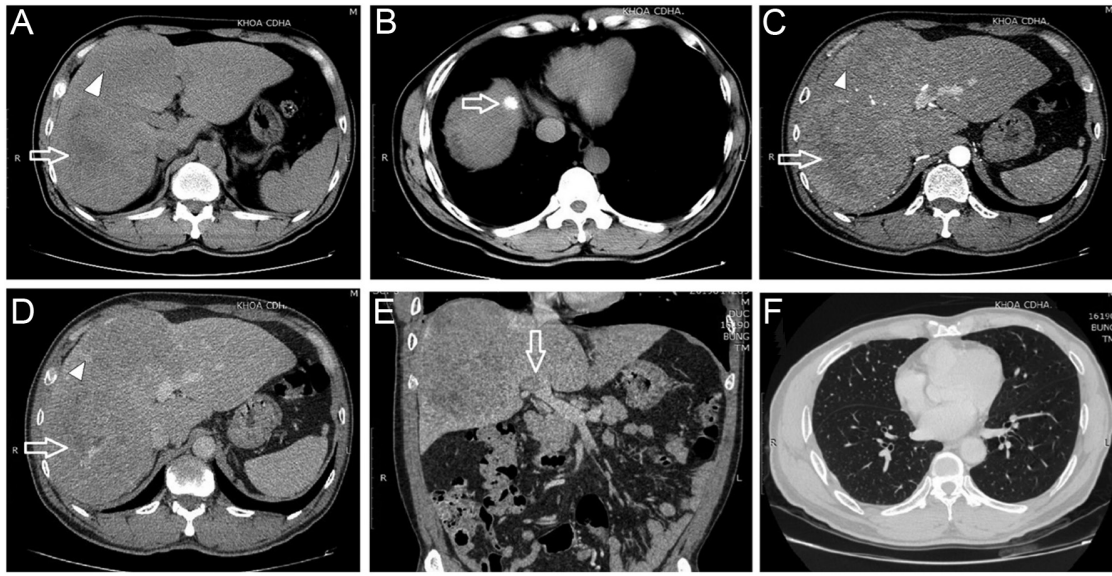

Figure 10

Abdominal and chest CT images obtained 15 months after the last check-up. (A) Axial, non-enhanced $C T$ image showing a hypoattenuating lesion in the fourth segment of the liver (white arrowhead) and most of the right lobe (white arrow), with an unclear boundary. (B) Axial, non-enhanced CT image showing a calcified mass in the liver (arrow). (C) Axial CT image in the arterial phase, showing the heterogeneous enhancement of the lesions (white arrow and white arrowhead). (D) Axial CT image in the venous phase, revealing lesions with signs of washout in the venous phase (white arrow and white arrowhead). (E) Coronal CT images in the venous phase, showing complete thrombosis of the right branch of the portal vein (white arrow). (F) No lesions were observed on thoracic CT.

Therefore, the follow-up of patients remains necessary to confirm the diagnosis. In this case, 15 months after the initial PHNET diagnosis was made, no extra-hepatic lesions were detected on abdominal and thoracic CT scans, confirming the diagnosis of PHNET.

Currently, no official, globally accepted treatment guide has been established for PHNET; however, surgery remains a popular treatment method due to the potential for a complete cure (16). According to Knox et al., the rate of PHNET resection is $92 \%$, and the 10 -year survival rate after liver resection is $68 \%$ (17). In patients who cannot be treated with surgery, various alternative treatment options are available, such as transarterial chemoembolization (TACE), chemotherapy or liver transplantation (18). As reported by Huang et al. (19), among patients who were either not eligible for surgery or experienced relapse and were treated with TACE, the 5-year survival rate reached $74-78 \%$. According to Park et al. (20), four of the twelve patients with multiple hepatic tumors who were treated by only chemotherapy survived for a median of 11.3 months (ranging from 3.0 to 26.4 months) (20). The effects of chemotherapy on PHNETs are not well known, although a number of studies have reported the use of a combination of drugs, such as 5-fluorouracil, doxorubicin, and streptozocin, for the treatment of non-surgical patients (20). In general, limited data exist regarding the treatment and prognosis of PHNET.

\section{Conclusion}

Primary hepatic neuroendocrine tumors are rare NET lesions found in the liver, characterized by nonspecific clinical and imaging results, which can be easily confused with other liver lesions, including
HCC and parasitic lesions. Therefore, the combination of a variety of diagnostic evaluation methods, such as imaging, gastrointestinal endoscopy, nuclear medicine, pathology, including histopathology, and immunohistochemistry, are necessary to provide a definitive diagnosis and classification. PHNET typically presents with medium differentiation and progresses slowly; therefore, if treated on time, surgery is often the most effective treatment. However, patients must be monitored for a long time after surgery to ensure the continued absence of additional NET lesions outside of the liver.

\section{Declaration of interest}

The authors declare that there is no conflict of interest that could be perceived as prejudicing the impartiality of the research reported.

\section{Funding}

This research did not receive any specific grant from any funding agency in the profit, commercial or not-for-profit sector.

\section{Patient consent}

Written informed consent has been obtained from the patient for publication of the case report and accompanying images.

\section{References}

1 Klimstra DS, Modlin IR, Coppola D, Lloyd RV \& Suster S. The pathologic classification of neuroendocrine tumors: a review of nomenclature, grading, and staging systems. Pancreas 201039 707-712. (https://doi.org/10.1097/MPA.0b013e3181ec124e)

2 Song JE, Kim BS \& Lee CH. Primary hepatic neuroendocrine tumor: a case report and literature review. World Journal of Clinical Cases 20164 243-247. (https://doi.org/10.12998/wjcc.v4.i8.243) 
3 Camargo ÉS, Viveiros Mde Mde M, Corrêa Neto IJ, Robles L \& Rezende MB. Primary hepatic carcinoid tumor: case report and literature review. Einstein 201412 505-508. (https://doi. org/10.1590/S1679-45082014RC2745)

4 Li W, Zhuang BW, Wang Z, Liao B, Hong LY, Xu M, Lin XN, Xie XY, Lu MD, Chen LD, et al. Case report of contrast-enhanced ultrasound features of primary hepatic neuroendocrine tumor: a CARE-compliant article. Medicine 201695 e3450. (https://doi. org/10.1097/MD.0000000000003450)

5 Kellock T, Tuong B, Harris AC \& Yoshida E. Diagnostic imaging of primary hepatic neuroendocrine tumors: a case and discussion of the literature. Case Reports in Radiology 20142014 156491. (https:// doi.org/10.1155/2014/156491)

6 Assarzadegan N \& Montgomery E. What is new in 2019 World Health Organization (WHO) classification of tumors of the digestive system: review of selected updates on neuroendocrine neoplasms, appendiceal tumors, and molecular testing. Archives of Pathology and Laboratory Medicine 2020145 664-677. (https://doi. org/10.5858/arpa.2019-0665-RA)

7 Baek SH, Yoon JH \& Kim KW. Primary hepatic neuroendocrine tumor: gadoxetic acid (Gd-EOB-DTPA)-enhanced magnetic resonance imaging. Acta Radiologica Short Reports 20132 2047981613482897. (https://doi.org/10.1177/2047981613482897)

8 Lin CW, Lai CH, Hsu CC, Hsu CT, Hsieh PM, Hung KC \& Chen YS Primary hepatic carcinoid tumor: a case report and review of the literature. Cases Journal 20092 90. (https://doi.org/10.1186/17571626-2-90)

9 Shi C, Zhao Q, Dai B, Xie F \& Yang J. Primary hepatic neuroendocrine neoplasm: long-time surgical outcome and prognosis. Medicine 201897 e11764. (https://doi.org/10.1097/ MD.0000000000011764)

10 Jia C, Zhang Y, Xu J \& Sun K. Experience in primary hepatic neuroendocrine tumor. Turkish Journal of Gastroenterology 201223 546-551. (https://doi.org/10.4318/tjg.2012.0370)

11 Quartey B. Primary hepatic neuroendocrine tumor: what do we know now? World Journal of Oncology 20112 209-216. (https://doi. org/10.4021/wjon341w)
12 Wang LX, Liu K, Lin GW \& Jiang T. Primary hepatic neuroendocrine tumors: comparing CT and MRI features with pathology. Cancer Imaging 201515 13. (https://doi.org/10.1186/s40644-015-0046-0)

13 Huang HF, Jin PP, Yang HJ, Zhang CJ, Zhang X, Wang JS, Yu JJ, Zhang B, Zhang Y \& Hu QD. Primary hepatic neuroendocrine tumor mimicking ruptured hepatocellular carcinoma with AFP elevation: a case report and literature review. OncoTargets and Therapy 202013 975-979. (https://doi.org/10.2147/OTT.S236728)

14 Skagias L, Vasou O, Ntinis A, Kondi-Pafiti A, Koureas A \& Politi E. Primary hepatic neuroendocrine tumor with exophytic growth: report of a case with diagnosis by fine needle aspiration biopsy. Acta cytologica 201054 202-204. (https://doi. org/10.1159/000325010)

15 Pan B, Wang SC, Chen ZK \& Zou GC. 18F-FDG-PET/CT findings of a primary hepatic neuroendocrine tumor: a case report and literature review. Journal of Clinical Case Studies 2019 5. (https://doi. org/10.16966/2471-4925.194)

16 Yalav O, Ülkü A, Akçam TA, Demiryürek H \& Doran F. Primary hepatic neuroendocrine tumor: five cases with different preoperative diagnoses. Turkish Journal of Gastroenterology 201223 272-278. (https://doi.org/10.4318/tjg.2012.0465)

17 Knox CD, Anderson CD, Lamps LW, Adkins RB \& Pinson CW. Long-term survival after resection for primary hepatic carcinoid tumor. Annals of Surgical Oncology 200310 1171-1175. (https://doi. org/10.1245/aso.2003.04.533)

18 Wängberg B, Nilsson O, Johanson VV, Kölby L, Forssell-Aronsson E, Andersson P, Fjälling M, Tisell L \& Ahlman H. Somatostatin receptors in the diagnosis and therapy of neuroendocrine tumor. Oncologist 19972 50-58. (https://doi.org/10.1634/theoncologist.2-1-50)

19 Huang YQ, Xu F, Yang JM \& Huang B. Primary hepatic neuroendocrine carcinoma: clinical analysis of 11 cases. Hepatobiliary and Pancreatic Diseases International 20109 44-48.

20 Park CH, Chung JW, Jang SJ, Chung MJ, Bang S, Park SW, Song SY, Chung JB \& Park JY. Clinical features and outcomes of primary hepatic neuroendocrine carcinomas. Journal of Gastroenterology and Hepatology 201227 1306-1311. (https://doi.org/10.1111/j.14401746.2012.07117.x)

Received in final form 14 January 2021 Accepted 13 May 2021 